\title{
Presentación:
}

\section{Resultados de los Procesos de Inclusión de Estudiantes Sordos y Estudiantes Ciegos en la Educación Regular}

\author{
Results of the Processes of Inclusion of Deaf Students and Blind \\ Students in Regular Education
}

\author{
Ricardo Rosas * \\ Pontificia Universidad Católica de Chile, Chile
}

Desde la declaración de Salamanca en 1994, la educación inclusiva ha sido un movimiento global. La declaración postula que las escuelas con una orientación inclusiva son el medio más efectivo para combatir actitudes discriminatorias, promover el establecimiento de comunidades acogedoras, construir una sociedad inclusiva y mejorar la eficiencia y el costo-efectividad del sistema educacional completo. A la fecha existe abundante evidencia, producto de estudios realizados mayoritariamente en países europeos o norteamericanos, que muestra resultados favorables de estas políticas con estudiantes en situación de discapacidad, particularmente física y cognitiva, tanto a nivel de indicadores académicos como socioemocionales. Sin embargo, en el caso particular de la discapacidad sensorial, hay poca evidencia de experiencias inclusivas con estudiantes sordos y estudiantes ciegos, especialmente en los países Latinoamericanos. Por consiguiente, la sección monográfica de este número está dedicada a recoger y difundir estudios relacionados con resultados académicos y socioemocionales de estudiantes ciegos o sordos en contextos inclusivos, desde la educación prebásica hasta la educación superior. Nos Interesaba recoger evidencia sobre cómo se han abordado las barreras que estos estudiantes enfrentan para acceder a la información visual o auditiva respectivamente, al aprendizaje y a la comunicación. En el caso de la comunidad sorda, es relevante conocer proyectos o innovaciones que abordan la inclusión entre sordos y oyentes desde un enfoque intercultural bilingüe, así como artículos que pongan en discusión los paradigmas y enfoques propuestos para la educación de las personas sordas, sus tensiones, resultados y desafíos.

El primer artículo, escrito por Dall’Asen y Gárate, es una revisión de literatura donde se comparan las políticas educativas orientadas a la inclusión de sordos en escuelas regulares en Chile y Brasil. Se realiza un análisis considerando el impacto social de la implementación de estas medidas en la escuela, considerando sobre todo la perspectiva de exclusión manifestada por los estudiantes sordos.

El segundo artículo, presentado por Pérez-Castro y Cruz-Cruz, describe los resultados de una investigación cualitativa enfocada en el análisis de las experiencias de inclusión y exclusión de un grupo de personas sordas usuarias de lengua de señas mexicana. Los resultados indican que las personas sordas deben enfrentar una serie de desafíos a lo largo de sus trayectorias educativas, lo que debiera ser considerado en el diseño de políticas públicas de largo plazo para disminuir el riesgo de exclusión.

*Contacto: rrosas@uc.cl 
Luego se presenta un artículo desarrollado por Rosas, Espinoza, Hohlberg e Infante, donde se describen los resultados de una investigación que compara, desde una perspectiva cuantitativa, los resultados de aprendizaje de un grupo de niños y niñas de desarrollo típico y con discapacidad sensorial. Los resultados indican la presencia de diferencias según el tipo de discapacidad y el sistema escolar al que pertenecen. Se plantea la necesidad de contar con espacios educativos flexibles que se adapten a las distintas necesidades de los estudiantes.

Posteriormente, se presenta un estudio cualitativo de las autoras Larrazábal, Palacios y Espinoza que analiza las experiencias de inclusión en escuelas regulares de un grupo de estudiantes sordos. Se problematizan los resultados en torno a la necesidad de contar con las condiciones necesarias para la inclusión de estudiantes sordos, considerando como elementos centrales de cambios tanto las prácticas docentes como las comunitarias.

El quinto artículo, desarrollado por Santa Cruz, Espinoza y Hohlberg, se centra en el tema de la presencia de problemas emocionales en niños y niñas de desarrollo típico y con discapacidad sensorial. Los resultados indican mayor presencia de problemas conductuales y emocionales en los estudiantes con discapacidad sensorial, existiendo mayor presencia de problemas internalizantes entre los niños con discapacidad visual y mayor presencia de problemas externalizantes entre los estudiantes con discapacidad auditiva.

A continuación, el artículo desarrollado por García, Farías, Reyes y Vásquez analiza la participación académica de estudiantes con discapacidad sensorial de estudiantes universitarios. Entre las problemáticas descritas se puede observar una menor tendencia a la finalización de sus estudios universitarios, un menor rendimiento general, principalmente en las carreras de Ciencias Naturales y Matemáticas. Las trayectorias escolares y el tipo de discapacidad son los principales factores facilitadores u obstaculizadores de la inclusión de estos estudiantes.

El séptimo artículo de la sección monográfica de este número de Palma-Picado, DelgadoAgüero y Moreira-Mora analiza la experiencia de un estudiante ciego y su desempeño en el ámbito de la Educación Superior, específicamente en una carrera de ingeniería. Se destaca la importancia de articular acciones y normativas vigentes para garantizar la inclusión de los estudiantes con discapacidad visual.

Por su parte, la sección de temática libre ofrece tres interesantes artículos que abordan distintas problemáticas relacionadas con la inclusión en educación. El trabajo de David Román, a partir de un enfoque poscrítico, analiza el fenómeno de la migración, sus características y aproximaciones conceptuales que permiten ahondar en su comprensión para la transformación de la escuela, considerando las necesidades de la diversidad del estudiantado como principio fundamental.

En segundo lugar, las autoras López y Manghi, a través de un estudio de casos múltiples, analizan la influencia del trabajo colaborativo en la transformación de las prácticas evaluativas desde una perspectiva inclusiva. Los hallazgos muestran una variedad de prácticas, destacando la adecuación de los instrumentos de evaluación y la retroalimentación.

Por último, el artículo de Santos Herrera estudia los significados y relevancia que las políticas educativas chilenas relativas a la inclusión le atribuyen al componente de la participación. Mediante el uso de procedimientos propios del análisis crítico del discurso, 
se corrobora una relegación de la noción de participación hacia un lugar destituido de su pretensión original como dinamizadora del proceso inclusivo.

Estamos seguros de que esta edición de la Revista Latinoamericana de Educación Inclusiva estimulará la investigación teórica y empírica que permita añadir nuevas evidencias, datos y perspectivas que hagan de nuestro sistema educacional uno cada vez más inclusivo.

\section{Breve CV del autor}

\section{Ricardo Rosas}

Psicólogo de la Pontificia Universidad Católica de Chile y Doctorado en Psicología Cognitiva de la Freie Universität Berlin. Profesor Titular de la Escuela de Psicología de la PUC. Sus áreas de interés son la Inteligencia, la Psicología de las Discapacidades, el Juego y Aprendizaje Implícito y el desarrollo de sistemas instruccionales basados en tecnologías. Es autor de cuatro libros y numerosas publicaciones en revistas internacionales en sus temas de investigación. Actualmente dirige el Centro de Desarrollo de Tecnologías de Inclusión de la PUC, CEDETi-UC que ha recibido dos importantes premios internacionales: WISE Award 2011 por el programa SUEÑALETRAS, diseñado para ayudar en la mediación de la lectura de niños sordos y el Premio Reina Letizia 2018, en la categoría Discapacidad y Rehabilitación. ORCID ID: https://orcid.org/0000-00023091-4044.Email: rrosas@uc.cl 